\title{
9.4
}

\section{Infrared Spectroscopy for the Detection of Adulteration in Foods}

\author{
Banu Özen and Figen Tokatli \\ Department of Food Engineering, İzmir Institute of Technology, Urla, İzmir, Turkey
}

\subsubsection{Introduction}

The use of infrared (IR) spectroscopy in food analysis dates back to the 1960s; the quantification of the fat, protein and lactose contents of milk was one of the first applications of mid-IR spectroscopy in the analysis food products [1]. Mid-IR spectroscopic analysis within food science and quality assurance was limited until the late 1980s due largely to difficulties in sample preparation and the presence of water, which absorbs strongly in the spectral region [2]. However, with improvements in the performance of infrared spectrometers and introduction of new mid-IR sampling techniques such as attenuated total reflectance (ATR) accessories in recent years the number of applications to the analysis of food has been steadily increasing. Developments in multivariate statistical analysis (chemometric) techniques have also helped IR spectroscopy to find many more applications in food science and the use of chemometrics has become an inseparable part of both mid-IR and near-IR (NIR) spectroscopic data analysis.

IR spectroscopic analysis of foods provides several advantages, including short analysis time, ability to measure multiple components in a single analysis, the need for only a small amount of waste chemicals, and the requirement of a small amount of sample. In addition, some techniques such as the ATR measurement require minimum sample preparation and are non-destructive.

IR spectroscopy has found many applications in studies related to the quality and process control of food products, the determination of chemical composition and checking the authenticity of foods. IR spectroscopy has become established as a tool that is very useful in predicting the quality control parameters of edible oils;

Infrared and Raman Spectroscopy in Forensic Science, First Edition. Edited by John M. Chalmers, Howell G.M. Edwards and Michael D. Hargreaves. (c) 2012 John Wiley \& Sons, Ltd. Published 2012 by John Wiley \& Sons, Ltd. 
the determination of the isolated trans bonds in fats and oils by ATR/IR spectroscopy was designated as an official method by the American Oil Chemists' Society [3, 4]. In addition, parameters such as free fatty acid, peroxide and iodine values could be predicted effectively from mid-IR spectra in conjunction with chemometric techniques [5-7]. Determination of methanol content during distillation of grape-derived beverages [8], measurement of glucose and ethanol content during baker's yeast fermentation [9], and monitoring of the enzymatic hydrolysis of starch for the production of sugar mixtures $[10,11]$ are some other examples of the wide range of uses of IR spectroscopy in food and beverage process and quality control. Detection of adulteration of food products is another key area where application of IR spectroscopy has been successful, and a publication by Vermeulen et al. [12] reviews the authentication and traceability of agricultural and food products using vibrational spectroscopy, particularly from a European perspective. The next section of this chapter focuses on the use of mid-IR spectroscopy for the authentication of food products.

\subsubsection{Adulteration of Food Products and Application of IR Spectroscopy in the Detection of Adulteration}

Although food products are mostly adulterated to gain economic profit, some added adulterants have caused serious health problems. Within the European Union (EU), legislations concerned with the adulteration of food products generally can be found under a labelling act and there are several EU legislations concerning the right labelling of food products (e.g., European Economic Community [EEC] Regulations: 1576/89 [13], 1601/ 91 [14], 2081/92 [15], 2200/96 [16], 2815/98 [17], 2082/92 [18]). One of these legislations (EEC Regulation 2081/92) introduced by the EU is the Protected Designation of Origin (PDO) legislation [19], which aims at encouraging diverse agricultural production, protecting product names from misuse and imitation, and helping consumers by giving them information concerning the specific characteristics of the product $[15,18]$. PDO means the product is produced, processed and prepared within the specified geographical area and the quality or characteristics of the product are "essentially due to that area". "Cilento" virgin olive oil is an example of a product whose origin is guaranteed and is defined as "PDO". Further, Protected Geographical Indication (PGI) means the product is produced, processed and prepared in the geographical area [19] and the quality, reputation or characteristics of the product are attributable to that area. Some of the cheeses such as Asiago, Bitto and Fontina and some olive oils produced in Greece, Spain, France, Italy and Portugal have PDOs. Piedmont hazelnut, Borgotaro mushrooms, Montella chestnut, Sicilian blood orange and Norcia ham are examples of products which have PGIs. According to the World Trade Organization Agreement on TradeRelated Aspects of Intellectual Property Rights (TRIPS) "geographical indications" is defined as indications that identify a product as originating in the territory of a Member, or a region or locality in that territory, where a given quality, reputation or other characteristic of the product is essentially attributable to its geographic origin [20].

As these legislations imply, adulteration is more than just mixing an adulterant with pure food and has become more complex to detect. Carcea et al. [21] categorised food adulteration into five groups by taking European legislation as a reference point:

- The misdescription of the name of food and non-compliance with the requirements of legal name (e.g., virgin olive oil, chocolate, jam, etc.);

- The adulteration of foods or substitution with lower value ingredients;

- The misdescription of geographical species, variety and production origin;

- The non-declaring of certain processes in the ingredients or preparation of food;

- Incorrect quantitative ingredient declarations. 
Most conventional adulteration detection methods rely on determination of a marker. However, use of a single marker may be misleading since food products might show variability depending on factors such as geographic location of cultivation and variety. However, IR spectroscopy provides information on multiple components, therefore, could be a powerful tool for adulteration detection.

Generally, adulteration detection regarding the geographical indication requires first building a database of the product by taking into consideration factors such as harvest/production year and variety. Classification with respect to pure and adulterated products can be performed using chemometric techniques such as principal component analysis (PCA). For the determination of adulterant concentration multivariate regression tools like partial least square (PLS) analysis produces successful results. There are various examples of the use of IR spectroscopy in determination of authenticity of high economic value food products such as olive oil, honey, wine and dairy products.

Olive oil is a product which is commonly adulterated by mixing with cheaper substitutes. These substitutes could be other edible oils, refined olive oil or olive oils from outside of the designated geographic region. In the 1980 s consumption of fraudulent oil sold as olive oil resulted in an outbreak known as "toxic oil syndrome". About 20000 people were affected from this condition and at least 300 deaths occurred. Rapeseed oil denatured with aniline that was intended for industrial use was designated as the cause of this case [22]. Both NIR and mid-IR spectroscopy were applied to the classification of olive oils with respect to their geographical origin. In general, satisfactory results were obtained for the classification of the samples according to their designation of origin [23-27]. These techniques are also very effective in determining the adulteration of olive oil with cheaper substitutes like hazelnut, sunflower, corn and soybean oils [28-30] and detection limits can be as low as $0.6 \%$.

Similar types of studies using both NIR spectroscopy [31-33] and ATR/mid-IR spectroscopy [34] have also been performed for wine. In each case, differentiation based on either geographic origin or grape variety was performed with IR spectroscopy in combination with multivariate data analysis. Classification of wines coming from different countries as well as different regions of the same country was studied. One of the factors affecting the rate of the success of the classification is the type of the statistical analysis method employed [31]. As in the case of oil adulteration, results of wine adulteration might be lethal. There are several reported cases of death through methanol poisoning due to consumption of methanol added to alcoholic beverages $[35,36]$. NIR spectroscopy was used to classify distilled alcoholic beverages (rum, whisky, brandy, vodka) and also to detect their adulteration with either one of ethanol, methanol or water at the 5-10\% level [37]. 100\% correct prediction in detection of adulteration was obtained for all cases and researchers proposed that the method could be used as a screening analysis to verify adulteration of the alcoholic beverages prior to more elaborate tests. Fourier transform infrared (FT-IR) spectroscopy was also used in detecting glycerol $(0.1-15.0 \%)$ in red wine and artificial neural networks and other chemometric techniques were used to process the data. PLS was used for quantitative analysis, while linear discriminant analysis (LDA) and canonical variate analysis (CVA) were used for classification and discrimination purposes. Each of the data analysis techniques had over $90 \%$ success in classification of samples either as adulterated or non-adulterated wine [38].

Another example of the serious health effects of consuming deceitful food products is the recent high-profile health scare related to melamine adulteration of milk and dairy products and resulting acute kidney failures and related fatalities among babies that had consumed these products. It has been reported that different forms of IR spectroscopy were used successfully in detecting the melamine contamination in infant formulas, powdered and liquid milk [39, 40]. NIR, ATR/FT-IR and diffuse reflection/FT-IR spectroscopy methods each achieved $1 \mathrm{ppm}$ level as the threshold of the detection for the infant baby formula case [39]. The threshold level obtained with these methods is lower than the $2.5 \mathrm{ppm}$ level set by the United States Federal and Drug Administration (FDA) as an amount that does not raise public concern in food products except for infant 
formulas. The threshold set for melamine in infant formulas was determined as $1 \mathrm{ppm}$ [41]. Extraction of melamine from milk samples with trichloroacetic acid and acetonitrile was performed before testing the samples with ATR/FT-IR spectroscopy; PLS together with mean centring mathematical enhancement was used as the statistical tool for the analysis of second derivative spectra by Wang et al. [40].

Dietary supplements are the products that could carry health and nutritional claims. Due to these claims consumers' interest in these types of products has been increasing lately. As a consequence, they have become very much prone to adulteration. There are several examples of IR spectroscopy studies for the detection of mixing dietary supplements with adulterants. It was reported that ginseng, an expensive herb, is adulterated with sawdust and Platycodon grandiflorum (jiegeng) and PCA analysis of FT-IR second derivative spectra was able to make a discrimination between the adulterants and ginseng [42]. Detection of adulteration of dietary supplement oils such as flaxseed, borage and evening primrose with cheaper alternatives was successfully achieved to levels as low as $2 \%$ with the use of mid-IR spectroscopy [43]. In another study, a new algorithm called "local straight-line screening" based on IR spectroscopy was developed to detect synthetic drugs as the adulterants of herbal medicines [44]. With this algorithm, the intention was to resolve complex IR spectra for better differentiation. This new approach was also tested with herbal medicine based diet pills and promising results were obtained using this algorithm suggesting it could be successfully applied on similar products. Modification of the same algorithm with interpolation and second derivatisation has provided improvement in detection of synthetic drugs in herbal medicines with IR spectroscopy and this technique was suggested to be a good tool for preliminary screening of herbal medicines [45].

In food analysis, mid-IR data are widely used to classify food samples (authentication based on geographical origin, variety) and to detect adulteration (e.g., in olive oil, honey). Multivariate statistical techniques are today almost an unavoidable part of spectroscopic data analysis, such as near- and mid-IR spectral profile datasets, in which one spectrum may contain intensity values for a thousand or more data points. PCA, soft independent modelling of class analogy (SIMCA), PLS regression and discriminant analysis (DA, PLS-DA) are among the common modelling techniques used for these purposes [46]. The techniques are based on mathematical and statistical operations on the data set, which usually has redundant information, so that the variance within the data can be explained by a few factors (e.g., principal components) rather than all the variables. If it is vibrational spectral data that have been recorded over many wavenumbers (or wavelengths), the absorbance or transmittance values at each will be the observations, and the wavenumbers (or wavelengths) will be the variables. The statistical analysis of data often includes the following procedures:

- Pre-treatment (e.g., normalisation, mean-centring, filtering-smoothing);

- Modelling (e.g., PCA, SIMCA, PLS, PLS-DA);

- Validation of model results with an independent data set.

Foods are complex chemical bodies. Their IR spectroscopic profiles carry too much information along with noise and baseline and path-length difference effects. Therefore, pre-treatment of data is usually a necessary task since it may enhance the model that is used to classify foodstuffs or to predict desirable/undesirable ingredients in them by eliminating or minimising irrelevant variances within the data.

\subsubsection{Case Study: Adulteration of Extra Virgin Olive Oils with Refined Hazelnut Oil}

Adulteration of extra virgin olive oil with hazelnut oil is very common due to their similar fatty acid profiles [12]. Therefore, it is a difficult task to determine the presence of hazelnut oil qualitatively and 
quantitatively, especially when it is present at low concentrations. The data used in a study in our laboratory were FT-IR spectra recorded from extra virgin olive oil samples adulterated with hazelnut oil in a range of $2-50 \%(\mathrm{v} / \mathrm{v})$ [47]. The instrument was equipped with a horizontal ATR sampling accessory (ZnSe crystal) and a deuterated tri-glycine sulfate (DTGS) detector. The resolution was set at $2 \mathrm{~cm}^{-1}$ and the number of scans collected for each spectrum was 128. Instead of using the whole spectral range between $4000-650 \mathrm{~cm}^{-1}$, two portions (3620-2520 and $1875.5-675.0 \mathrm{~cm}^{-1}$ ) were selected for the data analysis. The spectral data matrix has the dimension of $151 \times 4603$, with 151 spectra and 4603 data points per spectrum. The sample set contained pure olive oil and adulterated olive oil samples. For the evaluation of the model, the cross-validation [leave one out (LOO) cross-validation] was used to measure the prediction performance of the model. The result of this so-called inner validation process was expressed as a prediction coefficient of determination, $R^{2}$, value. In addition to this, an independent (validation) test data, which had not been used in the construction of the multivariate model, was analysed by the model to assess the general applicability of it. 21 samples were randomly selected from the sample set and used as a validation prediction data set and therefore not included in the modelling step. The root mean square error of calibration (RMSEC) and that of prediction (RMSEP) were determined for the test and validation data sets, in addition to $R^{2}$ values:

$$
\begin{gathered}
\text { RMSEC }=\sqrt{\frac{\sum_{i}^{m}\left(Y_{i}-\bar{Y}_{i}\right)^{2}}{m-a-1}} \\
\text { RMSEP }=\sqrt{\frac{\sum_{i}^{n}\left(Y_{i}-\bar{Y}_{i}\right)^{2}}{n}}
\end{gathered}
$$

where $m$ and $n$ are the number of observations in calibration and prediction (validation) sets, $a$ is the number of principal components of the model; $Y_{i}$ is the actual value of the quality variable $i$ and $\bar{Y}_{i}$ is its prediction. The data were auto-scaled and modelled with PLS regression to estimate the hazelnut oil concentration at $0-50 \%$; the hazelnut concentration in the sample is the $Y$ variable for this case study example. This yielded a four-component model with an $R^{2}$ of 0.959 for the calibration set and an $R^{2}$ of 0.938 for the validation set. RMSEC and RMSEP of the model were 3.508 and 3.954, respectively. Figure 9.4.1 a shows the scatter plot of predicted vs. actual concentrations. As can be seen, in this example, values close to the zero-percent levels (predicted as having values between -0.07 and $12.0 \%$ ) are those that especially degrade the precision of the model. Consequently, auto-scaled data were then filtered first with orthogonal signal correction (OSC) and digital wavelet analysis (Daubeshies-10) was applied before PLS analysis for the quantification of the adulterant. The OSC method is used in PLS modelling to remove systematically the information in spectral data ( $\mathbf{X}$ matrix) that is unrelated or orthogonal to the response variables ( $\mathbf{Y}$ matrix) [48]. OSC can be used as the single pre-treatment method or along with wavelet analysis. Wavelet analysis is used for denoising (smoothing) and compressing data in a series of procedures, where the original data are transformed from one numerical domain to another. The new compressed variables are the linear combination of the original ones $[49,50]$. This approach yielded a two-component model with an $R^{2}$ of 0.984 and $R^{2}$ prediction of 0.98. RMSEC and RMSEP of the model were 2.180 and 2.037, respectively. Figure 9.4.1b shows the predicted hazelnut percentages by the PLS model of OSC-wavelet treated spectra. The predictions are closer to the actual values and this time zero-percent sample predictions only extend over the range from -1.5 to $5.5 \%$. 


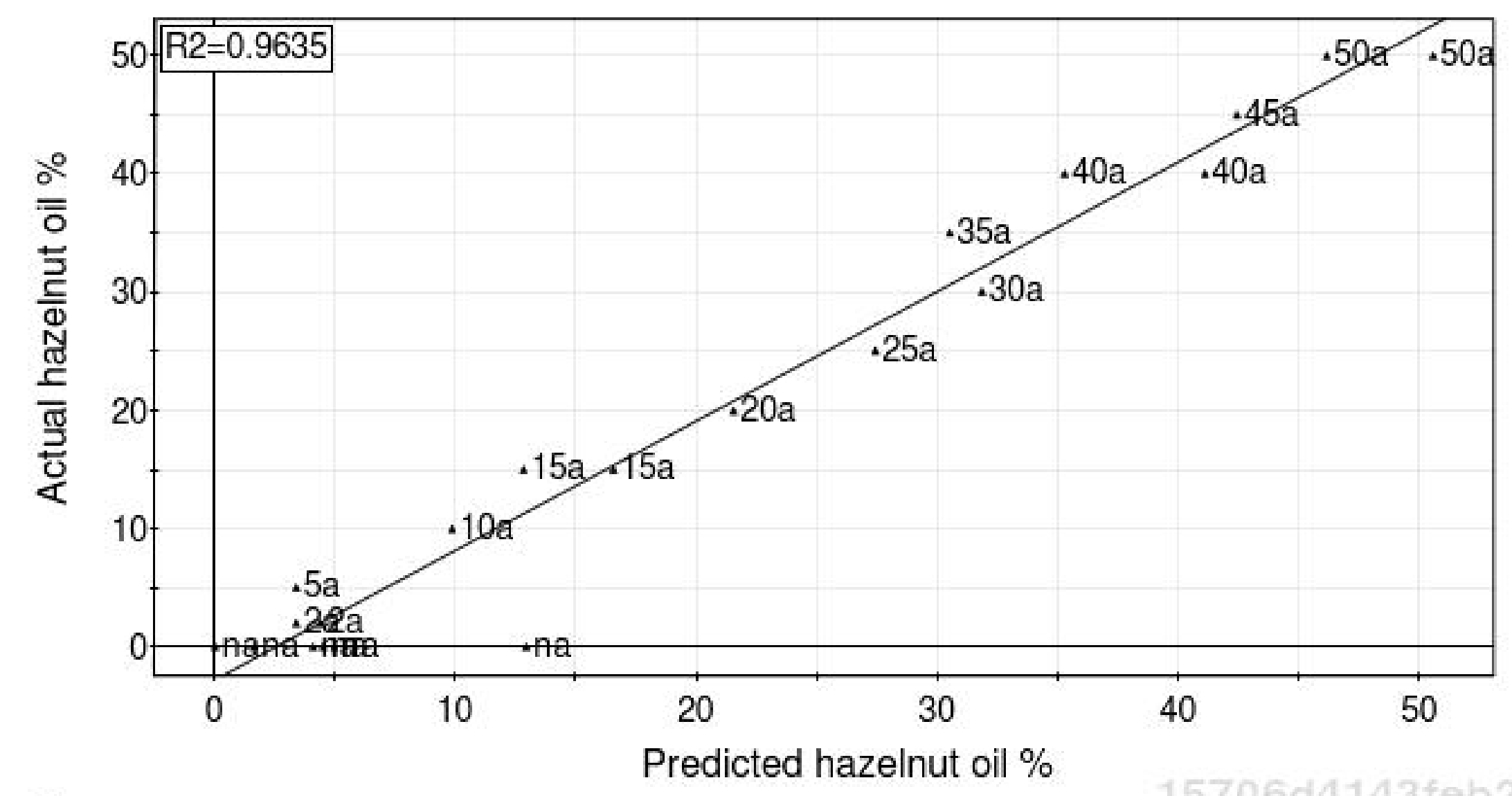

(a)

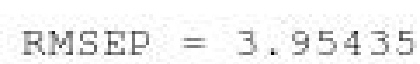

SIMCA-P $11.5-1 / 29 / 20102: 13: 52 \mathrm{PM}$

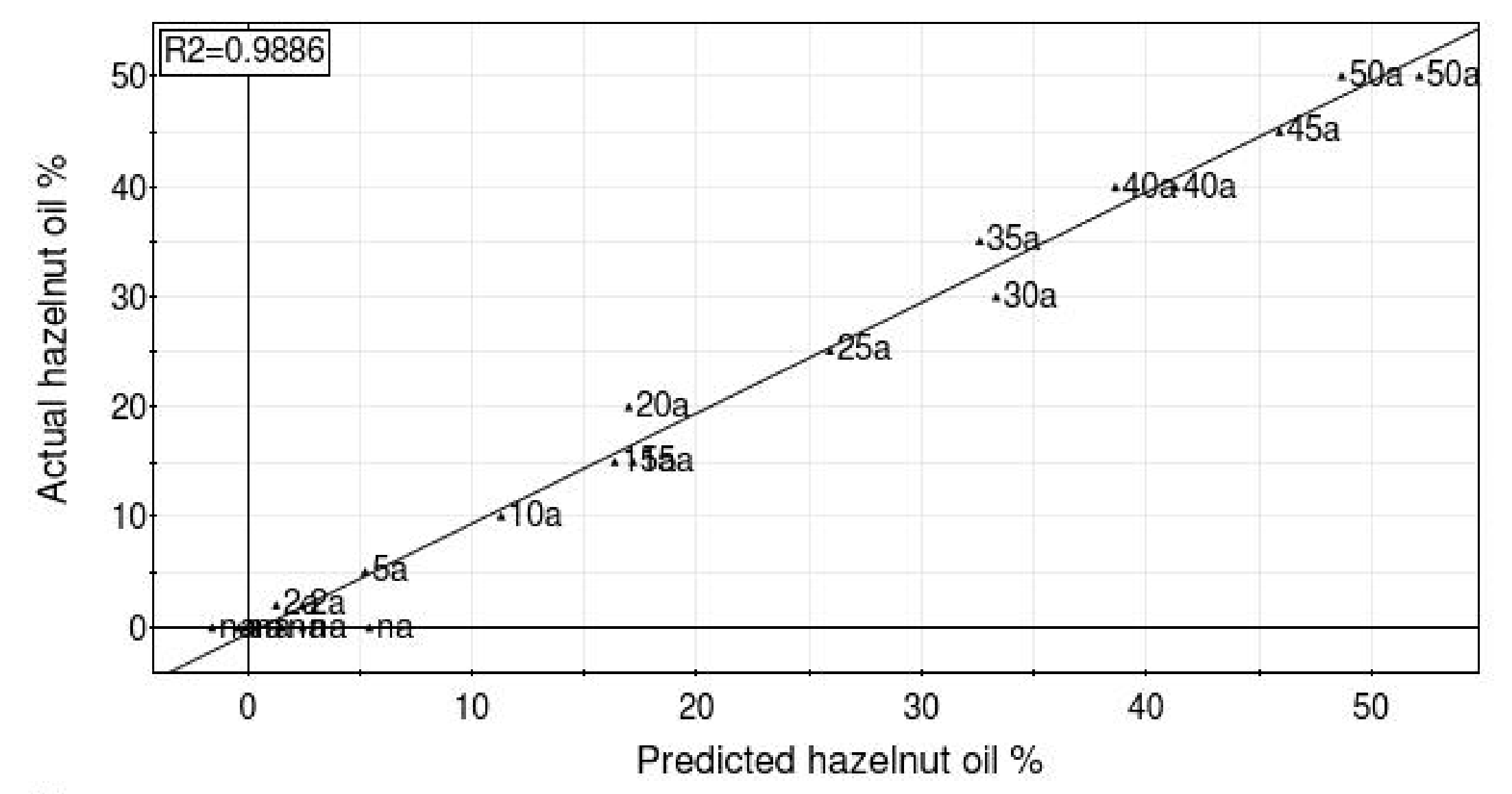

(b)

RMSEP $=2.03677$

SIMCA-P 11.5 - 1/29/2010 2:03:02 PM

Figure 9.4.1 (a) Results of PLS regression applied to prediction set of olive oil data. (b) Results of PLS regression applied to prediction set of OSC wavelet filtered olive oil data. na: Not adulterated. a: Adulterated at different percentages (2-50\% hazelnut oil).

PLS-DA analysis of the data is shown in Figure 9.4.2a as the scatter plot of the first two principal components. Here, it is difficult to say that the pure olive oil samples (class 1) are clearly separated from adulterated samples (class 2). Principal component plot of PLS-DA analysis of filtered data gives an improved discrimination between pure and mixed samples (Figure 9.4.2b). Evidently, in this example, the pre-treatment used of the data improved the classification ability of the models.

Further examples of the use of NIR and mid-IR and Raman spectroscopy coupled with a range of multivariate data analysis techniques can be found in Reference [12] and references cited therein, including the EU TRACE (tracing the origin of food) project [51]. 


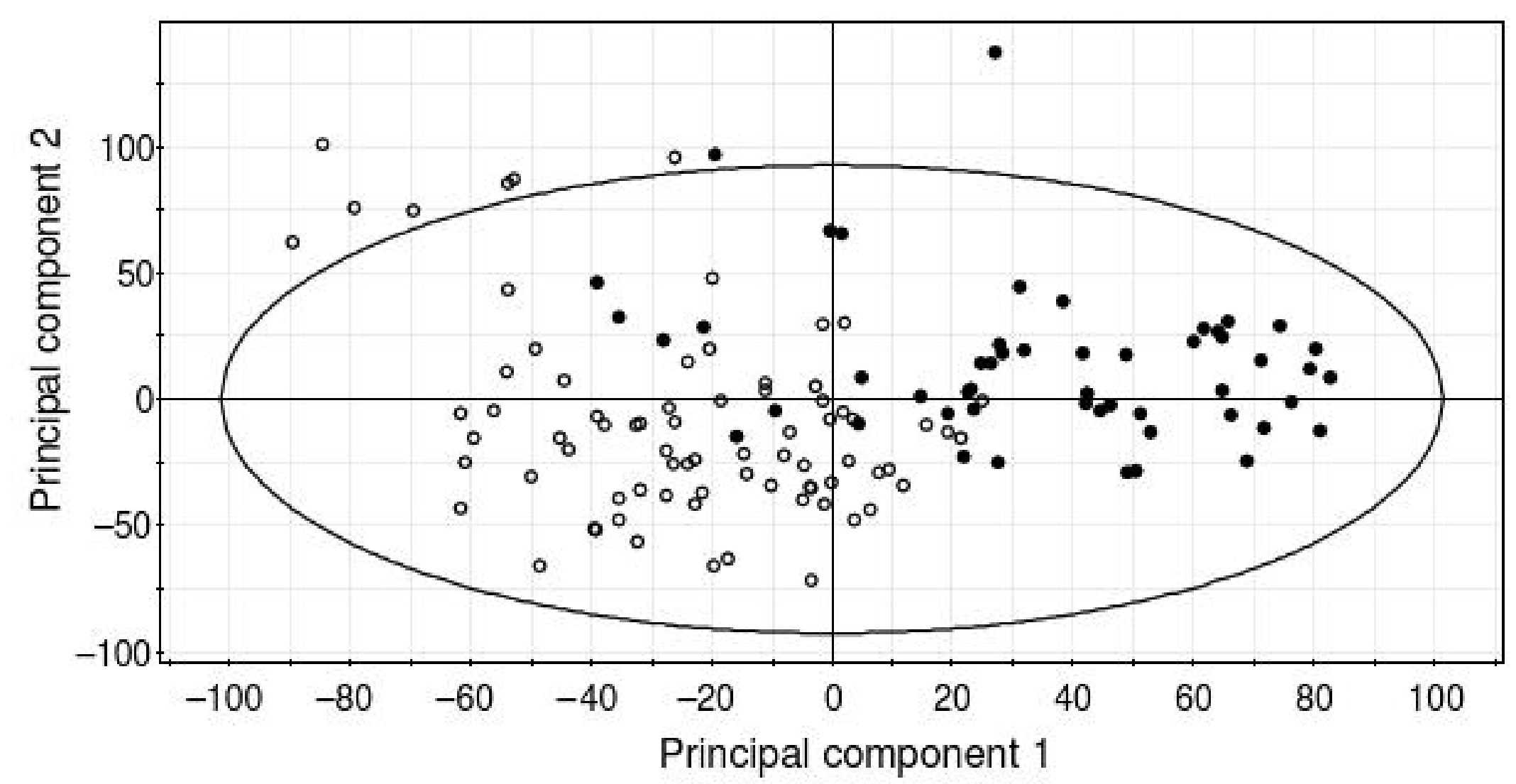

(a)

$-\quad$ Class $1 \quad$ Class 2
SIMCA-P $11.5-1 / 29 / 2010$ 3:03:34 PM

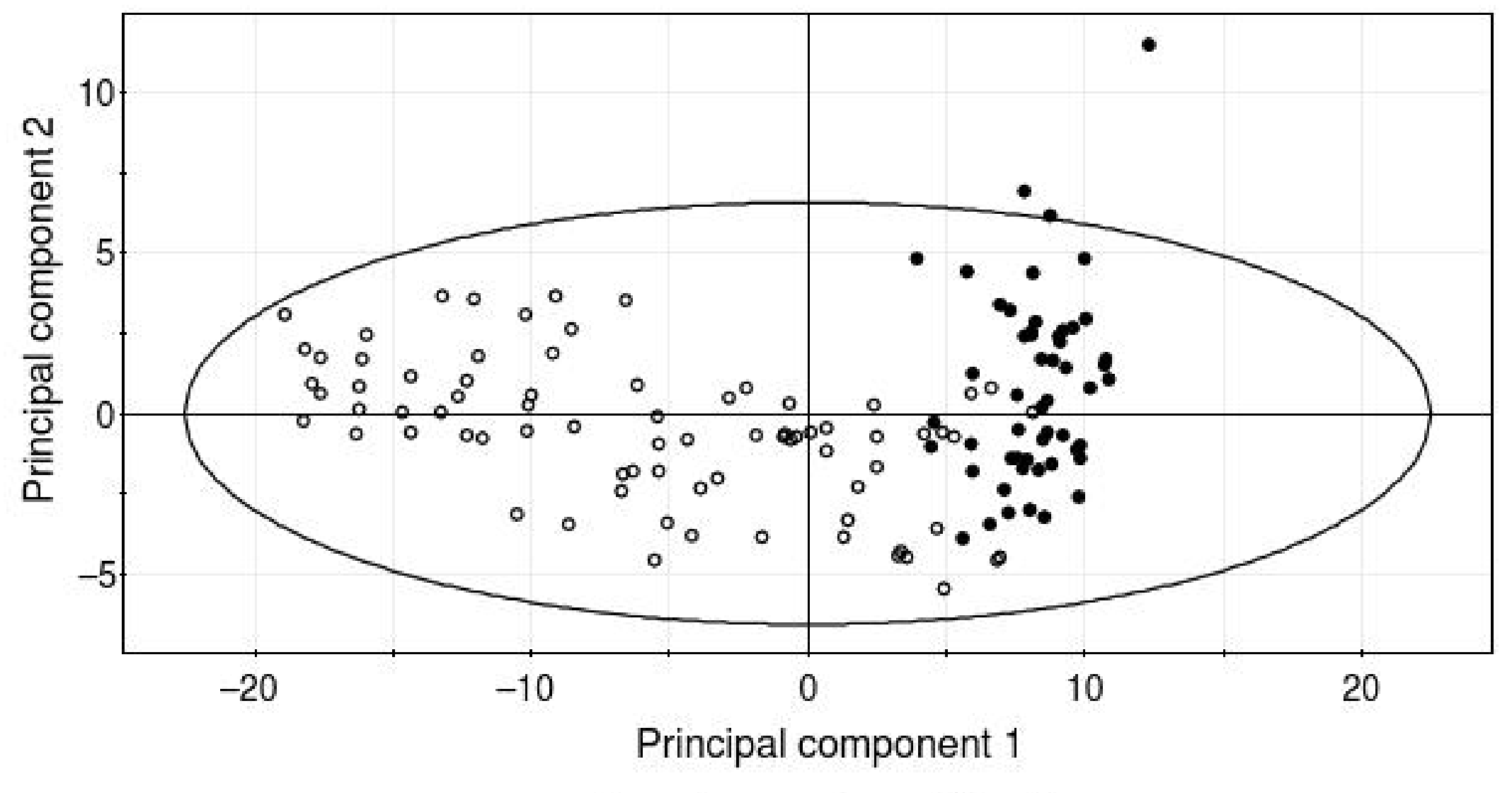

- Class 1 Class 2

(b)

Figure 9.4.2 (a) Score plot of PLS-DA model of olive oil data. (b) Score plot of PLS-DA model of OSC-waveletfiltered olive oil data. Class 1: pure olive oil samples. Class 2: olive oil/hazelnut oil mixtures.

\subsubsection{Summary}

IR spectroscopy in combination with chemometric techniques is an effective tool for the detection of adulteration of high economic value food products such as wine, dietary supplements and olive oil. It provides a practical and quick alternative to other commonly used analytical methods.

\section{References}

1. D.A. Biggs (1967) Milk composition: milk analysis with the infrared milk analyzer, J. Dairy Sci., 50, 799-803.

2. F.R. van de Voort, and A.A. Ismail (1991) Proximate analysis of foods by mid-FTIR spectroscopy, Trends Food Sci. Technol., 2, 13-17. 
3. American Oil Chemists Society (1988) Official Methods for the Determination of Trans Fat. Cd 14-95, AOCS, Chicago.

4. American Oil Chemists Society (1989) Official Methods for the Determination of trans Fat. Cd 14d-99, AOCS, Chicago.

5. A.A. Ismail, F.R. van de Voort, G. Emo and J. Sedman (1993) Rapid quantitative determination of free fatty acids in fats and oils by Fourier transform infrared spectroscopy, J. Am. Oil Chem. Soc., 70, 335-341.

6. J. Sedman, F.R. van de Voort, A.A., Ismail and P. Maes (1998) Industrial validation of Fourier transform infrared trans and iodine value analyses of fats and oils, J. Am. Oil Chem. Soc., 75, 33-39.

7. X. Yu, S. Du, F.R. van de Voort, T. Yue, and Z. Li (2009) Automated and simultaneous determination of free fatty acids and peroxide values in edible oils by FTIR spectroscopy using spectral reconstitution, Anal. Sci., 5, 627-632.

8. R.G. Dambergs, A. Kambouris, I.L. Francis, and M. Gishen (2002) Rapid analysis of methanol in grape-derived distillation products using near-infrared transmission spectroscopy, J. Agric. Food Chem., 50, 3079-3084.

9. G. Mazarevica, J. Diewok, J.R. Baena, E. Rosenberg, B. Lendl (2004) On-line fermentation monitoring by midinfrared spectroscopy, Appl. Spectrosc., 58, 804-810.

10. V. Bellon-Maurel, C. Vallat and D. Goffinet (1995) Quantitative analysis of individual sugars during starch hydrolysis by FT-IR/ATR spectrometry. Part I: multivariate calibration study-repeatability and reproducibility, Appl. Spectrosc., 49, 556-562.

11. M. Blanco, J. Coello, H. Iturriaga, S. Maspoch and R. G. Bano (2000) On-line monitoring of starch enzymatic hydrolysis by near infrared spectroscopy, Analyst, 125, 749-752.

12. Ph. Vermeulen, J.A. Fernández Pierna, O. Abbas, P. Dardenne and V. Baeten (2010) Authentification and traceability of agricultural and food products using vibrational spectroscopy, in Applications of Vibrational Spectroscopy in Food Science (eds E.C.Y. Li-Chan, J.M. Chalmers and P.R. Griffiths), John Wiley \& Sons Ltd, Chichester, ISBN: 978-0470-74299-0.

13. EEC (1989) Council Regulation No 1576/89 laying down general rules on the definition, description and presentation of spirit drinks, Official Journal, EC 1989, L160.

14. EEC (1991) Council Regulation (EEC) No 1601/91 laying down general rules on the definition, description and presentation of aromatized wines, aromatized wine-based drinks and aromatized wine-product cocktails, Official Journal, EC 1991, R1601-EN-01.01.2007-006.001-1.

15. EEC (1992) Council Regulation (EEC) No 2081/92 on the protection of geographical indications and designations of origin for agricultural products and foodstuffs, Official Journal, EC 1992, L208.

16. EC (1996) Council Regulation (EC) No 2200/96 on the common organization of the market in fruit and vegetables, Official Journal, EC 1996, R2200-EN-18.05.2001-002.001-1.

17. EC (1998) Commission Regulation (EC) No 2815/98 concerning marketing standards of olive oil, Official Journal, EC 1998, L349/56.

18. EEC (1992) Council Regulation (EEC) No 2082/92 on the specificity certification for agricultural products and foodstuffs, Official Journal, EC 1992, L208/9.

19. European Commission (2010) EU agricultural product quality policy, http://ec.europa.eu/agriculture/quality/ schemes/index_en.htm (accessed June 2010).

20. World Trade Organization (2010) TRIPS, http://www.wto.org/english/tratop_E/trips_e/gi_background_e.htm (accessed June 2010).

21. M. Carcea, P. Brereton, P. Hsu, S. Kelly, N. Marmiroli, F. Melini, C. Soukolis and D. Wenping (2009) Food authenticity assessment: ensuring compliance with food legislation and traceability requirements, Qual. Assur. Saf. Crops Food, 1, 93-100.

22. M. Posada, M. Castro, E. Kilbourne, R. Diaz-de-Rojas, I. Abaitua, J. Tabuenca and A. Vioque (1987) Toxic-oil syndrome: case reports associated with the ITH oil refinery in Sevilla, Food Chem. Toxicol., 25, 87-90.

23. O. Galtier, Y. Le Dréau, D. Ollivier, J. Kister, J. Artaud, and N. Dupuy (2008) Lipid compositions and French registered designations of origins of virgin olive oils predicted by chemometric analysis of mid-infrared spectra, Appl. Spectrosc., 62, 583-590.

24. M. Casale, C. Casolino, G. Ferrari and M. Forina (2008) Near infrared spectroscopy and class modelling techniques for the geographical authentication of Ligurian extra virgin olive oil, J. Near Infrared Spec., 16, 39-47. 
25. N. Sinelli, E. Casiraghi, D. Tura and G. Downey (2008) Characterisation and classification of Italian virgin olive oils by near- and mid-infrared spectroscopy, J. Near Infrared Spec., 16, 335-342.

26. G. Gurdeniz, B. Ozen and F. Tokatli (2008) Classification of Turkish olive oils with respect to cultivar, geographic origin and harvest year, using fatty acid profile and mid-IR spectroscopy, Eur. Food Res. Technol., 227, 1275-1281.

27. G. Downey, P. McIntyre, A. Davies and N. Antony (2003) Geographic classification of extra virgin olive oils from the Eastern Mediterranean by chemometric analysis of visible and near-infrared spectroscopic data, Appl. Spectrosc., 57, $158-163$.

28. A. Tay, R.K. Singh, S.S. Krishnan and J.P. Gore (2002) Authentication of olive oil adulterated with vegetable oils using Fourier transform infrared spectroscopy, Lebensm-Wiss. Technol., 35, 99-103.

29. B.F. Ozen and L.J. Mauer (2002) Detection of hazelnut oil adulteration using FT-IR spectroscopy, J. Agric. Food Chem., 50, 3898-3901.

30. A. A. Christy, S. Kasemsuran, Y. Du and Y. Ozaki (2004) The detection and quantification of adulteration in olive oil by near-infrared spectroscopy and chemometrics, Anal. Sci., 20, 935-940.

31. L. Liu, D. Cozzolino, W.U. Cynkar, M. Gishen and C.B. Colby (2006) Geographic classification of Spanish and Australian Tempranillo red wines by visible and near-infrared spectroscopy combined with multivariate analysis, $J$. Agric. Food Chem., 54, 6754-6759.

32. L. Liu, D. Cozzolino, W.U. Cynkar, R.G. Dambergs, L. Janik, B.K. O'Neill, C.B. Colby and M. Gishen (2008) Preliminary study on the application of visible-near infrared spectroscopy and chemometrics to classify Riesling wines from different countries, Food Chem., 106, 781-786.

33. W. Guggenbichler, C.W. Huck, A. Kobler, M. Popp and G.K. Bonn (2006) Near infrared spectroscopy, cluster and multivariate analysis - contributions to wine analysis, Journal of Food Agric. Environ., 4, 98-106.

34. P.A. Tarantilis, V.E. Troianou, C.S. Pappas, Y.S. Kotseridis and M.G. Polissiou (2008) Differentiation of Greek red wines on the basis of grape variety using attenuated total reflectance Fourier transform infrared spectroscopy, Food Chem., 111, 192-196.

35. M.K. Gülmen, D. Meral, A. Hilal, R. Akcan and N. Çekin (2006) Methanol intoxications in Adana, Turkey, Toxicol. Mech. Method, 16, 353-357.

36. N. Yaycı, H. Ağrıtmış, A. Turla and S. Koç (2003) Fatalities due to methyl alcohol intoxication in Turkey: an 8-year study, Forensic Sci. Int., 131, 36-41.

37. M.J.C. Pontes, S.R.B. Santos, M.C.U. Araujo, L.F. Almeida, R.A.C. Lima, E.N. Gaiao and U.T.C.P. Souto (2006) Classification of distilled alcoholic beverages and verification of adulteration by near infrared spectrometry, Food Res. Int., 39, 182-189.

38. V. Dixit, J.C. Tewari, B-.K. Cho and J.M.K. Irudayaraj (2005) Identification and quantification of industrial grade glycerol adulteration in red wine with Fourier transform infrared spectroscopy using chemometrics and artificial neural networks, Appl. Spectrosc., 59, 1553-1561.

39. L.J. Mauer, A.A. Chernyshova, A. Hiatt, A. Deering and R. Davis (2009) Melamine detection in infant formula powder using near- and mid-infrared spectroscopy, J. Agric. Food Chem., 57, 3974-3980.

40. J. Wang, S. Jun and Q.X. Li (2009) Rapid analysis of melamine content in powdered and liquid milk using Fourier transform infrared spectroscopy, Food Sci. Biotechnol., 18, 1199-1203.

41. U.S. Food and Drug Administration (2009) Melamine contamination in China, http://www.fda.gov/NewsEvents/ PublicHealthFocus/ucm179005.htm (accessed May 2010).

42. K.Y.-L. Yap, C.S.Lim, S.Y.Chan and Y.W.Chan (2006) Rapid differentiation of pharmaceutical bio molecules: ginseng and adulterants, International Conference on Biomedical and Pharmaceutical Engineering, ICBPE 2006, pp. 5-10.

43. B.F. Ozen, I. Weiss and L.J. Mauer (2003) Dietary supplement oil classification and detection of adulteration using Fourier transform infrared spectroscopy, J. Agric. Food Chem., 51, 5871-5876.

44. L. Feng, L. Shu, L. Jian, C. Guiliang, C. Yan, Q. Yunpeng, C. Yifeng and W. Yutian (2007) A new method for testing synthetic drugs adulterated in herbal medicines based on infrared spectroscopy, Anal. Chim. Acta, 589, 200-207.

45. Z. Xueyi, Z. Zhonghu, L. Geng, W. Yutian and Q. Yunpeng (2009) Modified local straight-line screening to detect synthetic drugs in adulterated herbal medicines, Appl. Spectrosc., 63, 471-476.

46. L. Eriksson, E. Johansson, N. Kettaneh-Wold and S. Wold (2001) Multi- and Megavariate Data Analysis: Principles and Applications, Umetrics Academy, Umea. 
47. G. Gurdeniz (2007) Chemometric Studies for Classification of Olive Oils and Detection of Adulteration, M.S. thesis, İzmir Institute of Technology, Urla-Izmir.

48. S. Wold, H. Antti, F. Lindgren and J. Ohman (1998) Orthogonal signal correction of near-infrared spectra, Chemical and Intelligent Laboratory Systems, 44, 175-185.

49. J. Trygg and S. Wold (1998) PLS regression on wavelet compressed spectra, Chemical and Intelligent Laboratory Systems, 42, 209-220.

50. X.Z. Wang (1999) Data Mining and Knowledge Discovery for Process Monitoring and Control, Springer, Heidelberg.

51. European Union (2010) TRACE project: Tracing the origin of food, http://www.trace.eu.org/index.php (accessed September 2010). 